\title{
Open conformation chromatin and pluripotency
}

\author{
Hitoshi Niwa ${ }^{1}$ \\ Laboratory for Pluripotent Cell Studies, RIKEN Center for Developmental Biology (CDB), Kobe, Hyogo 650-0047, Japan
}

Pluripotency, the ability of a cell to give rise to all of the cells of an organism, is a fascinating and mysterious characteristic of embryonic stem (ES) cells. Recent extensive molecular studies of mouse ES cells have revealed the roles played by transcription factor networks and epigenetic processes in the maintenance of ES cell pluripotency (Niwa 2007). However, no direct evidence to connect these two mechanisms had been reported until in the previous issue of Genes \& Development, in which Loh et al. (2007) presented new evidence concerning this missing link.

\section{Transcription factor network to maintain pluripotency}

Analysis of transcription factors expressed in ES cells in an undifferentiated state-specific manner has resulted in the identification of several key transcription factors involved in the maintenance of pluripotency. Of these, the POU family transcription factor Oct $3 / 4$ is regarded as pivotal because either its loss of function or its overexpression completely abolishes self-renewal and induces differentiation (Niwa 2007). The Sry-related transcription factor Sox 2 was first identified as a partner of Oct3/4 working to activate the transcription of target genes (Yuan et al. 1995). However, a recent report has indicated that the unique function of Sox 2 may be the indirect activation of Oct3/4 transcription (Masui et al. 2007). The NK2-class homeobox transcription factor Nanog is also regarded as a component of the core transcription factor network along with Oct $3 / 4$ and Sox 2 because it is required for the maintenance of pluripotency and has a dominant function in supporting the self-renewal of mouse ES cells in the absence of leukemia inhibitory factor (LIF), the essential cytokine in conventional mouse ES cell cultures (Chambers et al. 2003; Mitsui et al. 2003). Since these three genes possess binding sites for all three of these transcription factors, and the transcriptional activation of these genes by Oct $3 / 4$ and Sox 2 has been confirmed, it may be that they act as a selforganizing network that sustains the expression levels needed to maintain pluripotency (Niwa 2007).

${ }^{1}$ Correspondence.

E-MAIL niwa@cdb.riken.jp; FAX 81-78-306-1929.

Article is online at http://www.genesdev.org/cgi/doi/10.1101/gad.1615707.

\section{Epigenetic mechanism to maintain pluripotency}

In the past few years, several interesting findings concerning the epigenetic features of ES cells have been reported. The general abundance of transcriptionally active chromatin marks, such as trimethylation of Lys 4 of histone $\mathrm{H} 3$ (H3K4me3) and acetylation of histone $\mathrm{H} 4$ (H4Ac), has been noted (Azuara et al. 2006). In contrast, the histone marks associated with transcriptionally inactive genes, such as trimethylation of Lys 27 of histone H3 (H3K27me3), are generally more scarce, but are nonetheless enriched in specific regions of the genome (Bernstein et al. 2006). These regions, designated as the bivalent domains, are composed of areas containing short active and long silent histone marks, and frequently overlap the regions in which the binding sites for Oct3/4, Sox2, and Nanog are clustered (Boyer et al. 2005). For this reason, the bivalent domain is generally considered to function as a center that maintains pluripotency in undifferentiated cells by repressing the expression of genes activated immediately after the induction of differentiation. Additionally, polycomb-repressing complex 2 (PRC2) is regarded as a key regulator mediating the gene repression marks recruited to the bivalent domains by pluripotency-associated transcription factors (Lee et al. 2006). However, the functional significance of PRC2 in the maintenance of pluripotency should not be overestimated, because the loss of function of PRC2 components, such as Eed and Suz12, results in up-regulation of differentiation-associated genes but does not abolish self-renewal in mouse ES cells, indicating that their function is not essential to maintaining pluripotency (Montgomery et al. 2005; Pasini et al. 2007).

There have been no reports to date identifying the mechanisms by which ES cells are able to generate an open chromatin state at the genome-wide level, although there are several possible mechanisms for keeping chromatin in an open configuration. As the acetylation of histone $\mathrm{H} 3$ and $\mathrm{H} 4$ is important in transcriptional activation, both by promoting open chromatin structural rearrangements and by competing with silent histone marks such as di- and trimethylation of Lys 9 of histone H3 (H3K9me2 and H3K9me3), it follows that either the activation of histone acetyltransferases (HATs) or inactivation of histone deacetylases (HDACs) should contribute to the rearrangement of chromatin into an open 
conformation. Maintenance of $\mathrm{H} 3 \mathrm{~K} 4 \mathrm{me} 3$ by activation of a histone methyltransferase (HMTase) and/or inactivation of a histone demethylase (HDMase) specific to this modification would presumably result in keeping chromatin open. It was recently reported that unmethylated H3K4 is specifically recognized by a member of the DNA methyltransferase (Dnmt) family, Dnmt31 (Ooi et al. 2007). Dnmt3l lacks the activity of other Dnmts, but it recruits Dnmt3a, which functions to activate de novo methylation of DNA. DNA methylation is recognized by methyl DNA-binding proteins, such as MeCP2 and $\mathrm{MBD} 1$, and triggers the formation of heterochromatin by recruiting heterochromatin proteins $(\mathrm{HP})$. Since ES cells express high levels of both Dnmt31 and Dnmt3a, this mechanism should be countered by proteins that would maintain the methylation of H3K4 in order to keep chromatin in its open configuration. Conversely, exclusion of repressive histone marks-such as $\mathrm{H} 3 \mathrm{~K} 9 \mathrm{me} 2$, $\mathrm{H} 3 \mathrm{~K} 9 \mathrm{me} 3$, and $\mathrm{H} 3 \mathrm{~K} 27 \mathrm{me} 3$, which recruit HPs to form heterochromatin by activating HDM and/or inactivating HMTs specific for these modifications-represents a possible mechanism for generating open chromatin in ES cells.

Until recently, the enzymatic activity of a HDMase in the demethylation of a methylatd histone lysine was only identified in the LSD protein, which removes H3K4me1 and H3K4me2 (Klose and Zhang 2007). However, in a breakthrough finding reported in 2006, Tsukada et al. (2006) used a biochemical approach to purify a robust HDMase activity with specificity toward Lys 36 of histone H3 from mammalian cells, and identified Jumonji-C (JmjC) domain-containing histone demethylase 1A (JHDM1A) as the molecule responsible for this HDMase activity. Using the same strategy, a novel H3K9-specific HDMase was identified as JHDM2A (Yamane et al. 2006). The JmjC domain was then to be the catalytic domain of HDMase (Klose and Zhang 2007). Jumonji (abbreviated Jmj) means "cruciform" in Japanese, in reference to the morphological phenotype of Jmj mutant mice, in which the normal neural groove is crossed by abnormal grooves on the neural plates (Takeuchi et al. 1995). There are multiple genes containing JmjC domains in the mouse genome, and JHDM3/JMJD2 enzymes have been identified as HDMases that preferentially demethylate H3K9me3, H3K9me2, H3K36me3, and $\mathrm{H} 3 \mathrm{~K} 36 \mathrm{me} 2$ (Klose et al. 2006), although their functions in ES cells have not yet been assessed.

\section{JmjC members cross genetic and epigenetic mechanisms to maintain pluripotency}

Some researchers have emphasized the importance of genetic mechanisms in maintaining pluripotency, while others have suggested that epigenetic control plays the dominant role, creating something of a chicken-and-egg impasse. However, a recent discovery of great importance has provided a clue to solve this puzzle. Takahashi and Yamanaka (2006) found that transfection of four transcription factor genes (Oct3/4, Sox2, Klf4, and Myc) allows the establishment of pluripotency in mouse fibro- blast cells. In the resulting cells, which Takahashi and Yamanaka (2006) designated induced pluripotent stem (iPS) cells, the global pattern of histone marks is switched from the somatic cell pattern to the pluripotent cell pattern (Maherali et al. 2007), indicating that the transduction of the four transcription factors is able to change both the genetic and the epigenetic states, and that the genetic mechanism takes precedence in this context. Of these four transcription factors, Myc is known to be directly susceptible to epigenetic change (Yamanaka 2007). Myc is a well-known oncogene that binds to a specific DNA sequence as a transcriptional activator of specific target genes, and also functions as a mediator of DNA replication. It has been reported that one of the target genes of Myc is Gcn5, which encodes a $\mathrm{HAT}$, and that its transcriptional activation is critical to mediate the function of Myc in promoting cell proliferation (Knoepfler et al. 2006). This is in accordance with a mechanism for promoting the open chromatin structure needed to establish and maintain pluripotency, proposed above. However, Gcn5 function is not essential for early development, and Gcn5-null ES cells can be established (Lin et al. 2007), suggesting that it does not provide the sole mechanism at work in keeping pluripotent chromatin in an open conformation.

But what of the roles of the other three transcription factors in modulating the epigenetics of pluripotent cells? Since Sox2 and Klf4 function cooperatively with Oct3/4 (Nakatake et al. 2006), it seems evident that research should focus on elucidating the function of Oct3/4, the pivotal gene in this particular network. Lists of the target genes of Oct3/4 have been developed using a variety of approaches (Boyer et al. 2005; Loh et al. 2006; Matoba et al. 2006). The comparison of global gene expression profiling with cDNA microarrays of ES cells after repression of Oct3/4 and chromatin immunoprecipitation (ChIP), in combination with a genome-wide location technique for Oct3/4, yielded 372 putative primary target genes, which contain several genes encoding epigenetic modifiers (Matoba et al. 2006). From these data, in the previous issue of Genes \& Development, Loh et al. (2007) identified the two JmjC family members encoding HDMases, Imjd1a and Imjd2c, as Oct3/4 targets. They first identified clusters of Oct3/4-binding sites by ChIP-paired end ditag analysis (ChIP-PET) and confirmed their functional significance for transcriptional activation by reporter assay. Depletion of Imjd1a by small interfering RNA (siRNA) in ES cells resulted in the induction of differentiation with a global increase in H3K9me2, whereas depletion of $I m j d 2 c$ induced differentiation with a global increase in $\mathrm{H} 3 \mathrm{~K} 9 \mathrm{me} 3$, confirming that their HDMase activity is linked to the maintenance of pluripotency. They next identified the genes whose expression was affected by depletion of these JmjC genes by cDNA microarray analyses. One of the genes downregulated after depletion of Imjd1a is Tcl1, which encodes the adaptor protein of Akt (Thymoma viral protooncogene 1) that enhances its enzymatic activity (Teitell 2005). Akt1 is a key kinase in the Ras/PI3K/Akt1 signaling pathway that is important for propagation of mouse 
ES cells. It has been reported that Tcl1 is expressed in ES cells in a stem cell-specific manner, and that its promoter activity is positively regulated by Oct3/4 (Matoba et al. 2006). Moreover, knockout of Tcll in mouse embryos leads to preimplantation lethality (Narducci et al. 2002), and knockdown of Tcll in ES cells results in either the induction of differentiation or the repression of proliferation (Ivanova et al. 2006; Matoba et al. 2006). Loh et al. (2007) found that the level of H3K9me2 and H3K9me3 in the Tcl1 promoter increased in Jmjdla knockdown ES cells with a reduction in Oct $3 / 4$ recruitment, and that Jmjdla proteins are recruited to this promoter, indicating that removal of $\mathrm{H} 3 \mathrm{~K} 9 \mathrm{me} 2$ from the promoter by Jmjd1a is required for proper Oct3/4-dependent transcriptional activation of Tcl1 in ES cells. Interestingly, Tcl1 is functionally significant as the target of Jmjdla, since the ectopic expression of Tcl1 prevents the differentiation of ES cells induced by the depletion of Jmjdla. They identified Nanog as the Jmjd2c target and confirmed the recruitment of Jmjd2c to the Nanog promoter. Depletion of $J m j d 2 c$ resulted in an increase of $\mathrm{H} 3 \mathrm{~K} 9 \mathrm{me} 3$ with enrichment of HP1 $\beta$, but left recruitment of Oct3/4 unaffected. Nanog also has functional significance as the target of Jmjd2c, because the ectopic expression of Nanog prevents differentiation of ES cells induced by depletion of $J m j d 2 c$. These findings show that Oct3/4 activates transcription of target genes either directly as a transcriptional activator or indirectly by transcriptional activation of chromatin modifiers that keep target sites in an accessible chromatin conformation.

\section{Is pluripotency the default state?}

This important result provides the missing link between genetic and epigenetic mechanisms in the establishment and maintenance of pluripotency. As a genetic mechanism, a self-organizing network of transcription factors prevents differentiation and promotes proliferation, enabling the continuous self-renewal of ES cells. The epigenetic processes should be under the control of the pluripotent transcription factor network, functioning to keep the target genes of these transcription factors accessible and thereby allowing the self-organizing network to be maintained. The existence of such a positive feedback loop involving genetic and epigenetic systems has been hypothesized but never conclusively shown. The direct transcriptional regulation of Imjd1a and Imjd2c by Oct3/4 is the first evidence to support this model. Oct3/4, a key component of the pluripotent transcription factor network, activates transcription of HDMase genes, and these gene products are recruited to the target genes of Oct3/4 to maintain accessibility. Since one of the target genes of Jmjd2c is Nanog, which is also an important component of the pluripotent transcription factor network and is supposed to contribute to the transcriptional activation of Oct3/4, here the positive feedback loop is closed. During the establishment of iPS cells, Myc might enhance the accessibility of target genes for the other three transcription factors by stimulating DNA replication and/or transcriptional activation of Gcn5- encoding HAT. Once Oct3/4 has the chance to activate $I m j d 1 a$ and $I m j d 2 c$, these gene products manifest their HDMase activity by removing $\mathrm{H} 3 \mathrm{~K} 9 \mathrm{me} 2$ and H3K9me3 from target genes to allow activation by Oct $3 / 4$. Activation of the target genes of Oct3/4, including Nanog, promotes the establishment of the pluripotent transcription factor network, ultimately resulting in the establishment of pluripotency in somatic cell nuclei. Of course, to test this model, the role of these JmjC family genes in the generation of iPS cells should be examined.

It is now possible to propose that the pluripotent state is a default state of genetic systems (Fig. 1). The transcription factor network to determine pluripotency might be self-organized by a positive feedback mechanism, and this also might be true for other transcription factor networks that confer differentiated phenotypes. Therefore, if all genetic information is freed from epigenetic repression, all transcription factors begin to establish their preferred network via self-activation. However, in such a scenario, the networks must be mutually exclusive in order to give rise to a single phenotype in any given cell. If exclusion occurs simply by chance, all phenotypes would arise with equal frequency. That said, it remains possible that one transcription factor network is extremely stable due to the extraordinary power of its positive feedback and its ability to repress other networks. In the first differentiation event in mouse development, trophectoderm is segregated from the pluripotent cell lineage via competition between two transcription factors-Oct3/4 and Cdx2 (Niwa et al. 2005). These two transcription factors possess positive feedback loops that activate their own expression, respectively, and a complex formed by the two factors competes against the activation of both. In morula-stage embryos, Cdx 2 might exclude Oct3/4 via a signal from polarized epithelium, while the opposite may occur in the inner cells lacking such a polarity signal, suggesting that pluripotency is dominant in the default state. The dominant character of the pluripotent network has been shown by cell fusion experiments. When pluripotent stem cells are fused to differentiated cells, the resulting tetraploid cells tend to acquire pluripotency (Tada et al. 2001). Therefore, the ability of the pluripotent transcription factor network to promote an epigenetic mechanism to keep chromatin open may serve as an element of the positive feedback mechanism that maintains the network. This may be the reason why pluripotency can be established in somatic cell nuclei more easily than had been expected previously.

\section{Further genetics of the epigenetic mechanism in ES cells}

Activation of JmjC by Oct3/4 might not be the sole mechanism linking genetic and epigenetic systems. As mentioned above, there are several mechanisms other than the removal of silent histone marks for maintaining euchromaticity, and the introduction of histone marks for active transcription-e.g., H3K4me3-might be especially important. Methylation of H3K4 is introduced by 


\section{pluripotent state}

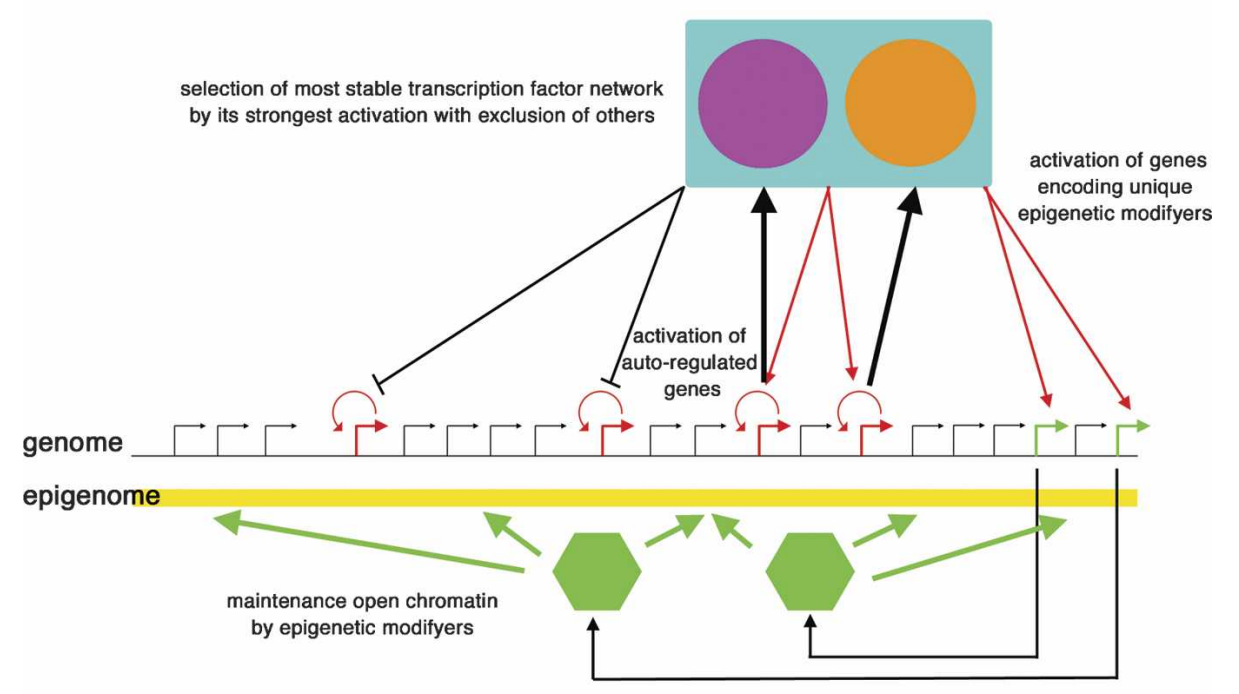

\section{differentiated state}

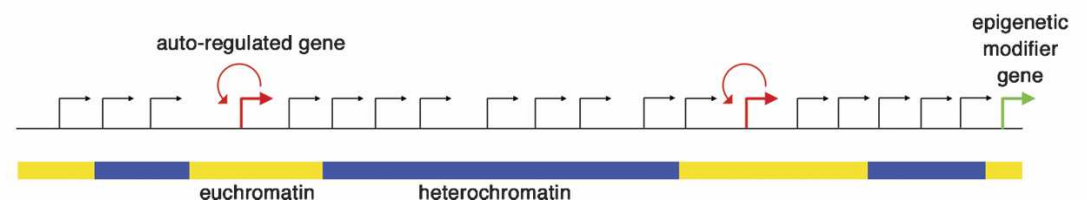

Figure 1. A model of the default pluripotent transcription factor network that arises from open chromatin. The unique gene expression profile specific to a particular differentiation state is thought to be generated by a combination of genetic (the presence of a set of transcription factors that form a self-organizing network) and epigenetic (regulation of access to specific genetic sequences by the transcription factors) factors, as shown for the differentiated state. In the pluripotent state, most of the epigenetic repression of genetic information is removed, resulting in the allowance of basal transcription for virtually all genes. In this situation, genes exhibiting positive feedback regulation-i.e., those encoding transcription factors that activate their own promoters-become activated. Competition between such genes ensues, resulting in the selection of the most potent and stable transcription factor network. When this transcription factor network has the ability to activate the transcription of genes encoding epigenetic modifiers and thereby to keep chromatin open, the characteristic pluripotent epigenome is stable in conjunction with the pluripotent transcriptome.

the SET domain-containing proteins belonging to the trithorax group, which contains six members (SETla, SET1b, MLL1-4) in the mouse genome (Glaser et al. 2006). Knockout studies in ES cells have been reported for Mll1 and Mll2, but no obvious phenotype has been found (Ernst et al. 2004; Glaser et al. 2006). Since the global level of H3K4me3 was not altered in these knockout ES cells, it appears that there may be other genes responsible for the trimethylation of H3 Lys 4 in ES cells. It was recently reported that Mll2 interacts with UTX, the JmjC family member that specifically demethylates H3K27me3 (Issaeva et al. 2007). The coupling of H3K4 methylation and H3K27me3 demethylation should have an important role in establishing the bivalent domain as well as its regulation during differentiation. Interestingly, in contrast to $\operatorname{Imj} j \mathrm{~d} 1 \mathrm{a}$ and $\operatorname{Imj} j 2 \mathrm{C}$, $\operatorname{Jm} j d 3$ encodes a H3K27me3 HDM (Agger et al. 2007; Lan et al. 2007), and is up-regulated after repression of Oct3/4 in ES cells (Matoba et al. 2006). This suggests that differential HDM activities might be coordinated by the pluripotent transcription factor network.
High levels of chromatin remodeling activity might also contribute to keeping chromatin open. The essential role of Snf2b (Brg1, Smarca4)-encoding a core component of the SWI/SNF and ISWI family remodeling complex-in the self-renewal of F9 embryonal carcinoma cells has been reported (Sumi-Ichinose et al. 1997), and Smarcad1 belongs to the SNF2 family of DEAD/H-boxcontaining helicase superfamily and was identified as a putative primary target of Oct3/4 (Matoba et al. 2006). The NuRD (nucleosome remodeling and histone deacetylation) complex component Mbd3 is required for the differentiation of ES cells, and it functions in the inner cell mass of the blastocyst to expand into ES cells in vitro (Kaji et al. 2007). However, most of these genes are expressed ubiquitously, and careful analysis will be required to distinguish the functions specific to the maintenance of pluripotency from other housekeeping functions.

The hyperdynamics of architectural chromatin proteins has been pointed out as a unique characteristic of ES cells, and the nucleosome assembly factor HirA was 
proposed as one of the factors responsible for this feature, since HirA-null ES cells exhibit elevated levels of unbound histones and the formation of embryoid bodies is accelerated in these mutant cells (Meshorer et al. 2006). In contrast to HirA, which acts as a chaperone of histone H3.3 in a DNA synthesis-independent manner, CAF1 acts as a chaperone of H3.1 in a DNA synthesis-dependent manner. It was recently reported that CAF1-null mouse embryos die at the 16-cell stage, suggesting it has an essential function in pluripotent cell populations (Houlard et al. 2006). In addition, a novel epigenetic feature of ES cells was revealed by Szutorisz et al. (2006), who showed that the proteasome acts on specific regulatory regions in ES cells to prevent incorrect transcriptional initiation to restrict permissive transcriptional activity while keeping the genes in a potentiated state. This system might contribute to keeping the threshold level of the promoter activity high for significant gene expression, thereby allowing the exclusive activation of the pluripotent transcription factor network, a hypothesis that has yet to be functionally confirmed.

For all epigenetic mechanisms, an important unsolved question remains. It has been clearly shown that Jmjd1a and Jmjd2c do not remove methylation of H3K9 ubiquitously, but that they are instead recruited to specific target genes to modulate local chromatin structure. This target-specific function has been observed in the proteasome. Since proteins there lack the ability to recognize specific DNA sequences, they should be recruited to the target genes by interaction with transcription factors binding a specific sequence. But which sequence-specific transcription factors work to recruit them? One possible and interesting candidate is none other than Oct3/4. Oct3/4 works as a transcriptional activator via interaction with multiple transcription factors in a context-dependent manner. In the case of PRC2, the role of Oct3/4 in recruiting it to target sites was suggested by the overlap of their binding sites in the genome (Lee et al. 2006). Oct3/4 cooperates with Sox 2 in several cases, and with both Sox2 and Klf4 on a subset of target genes. In addition, cooperation with factor(s) other than Sox2 is suspected for the activation of the Zfp 42 and Tcl1 promoters. Oct3/4 may recruit different JmjC family members in a cofactor-dependent manner. If so, the stability of the positive feedback mechanism, including both genetic and epigenetic factors, would be enhanced significantly. The solution of this puzzle will doubtless contribute to improving the efficiency of methods for establishing iPS cells, which may one day be used in the development of patient-specific cell therapies.

\section{Acknowledgments}

I thank Douglas Sipp (RIKEN CDB) for critical reading of the manuscript.

\section{References}

Agger, K., Cloos, P.A.C., Christensen, J., Pasini, D., Rose, S., Rappsilber, J., Issaeva, I., Canaani, E., Salcini, A.E., and He- lin, K. 2007. UTX and JMJD3 are histone H3K27 demethylases involved in HOX gene regulation and development. $\mathrm{Na}$ ture doi: 10.1038/nature06145.

Azuara, V., Perry, P., Sauer, S., Spivakov, M., Jorgensen, H.F., John, R.M., Gouti, M., Casanova, M., Warnes, G., Merkenschlager, M., et al. 2006. Chromatin signatures of pluripotent cell lines. Nat. Cell Biol. 8: 532-538.

Bernstein, B.E., Mikkelsen, T.S., Xie, X., Kamal, M., Huebert, D.J., Cuff, J., Fry, B., Meissner, A., Wernig, M., Plath, K., et al. 2006. A bivalent chromatin structure marks key developmental genes in embryonic stem cells. Cell 125: 315-326.

Boyer, L.A., Lee, T.I., Cole, M.F., Johnstone, S.E., Levine, S.S., Zucker, J.P., Guenther, M.G., Kumar, R.M., Murray, H.L., Jenner, R.G., et al. 2005. Core transcriptional regulatory circuitry in human embryonic stem cells. Cell 122: 947-956.

Chambers, I., Colby, D., Robertson, M., Nichols, J., Lee, S., Tweedie, S., and Smith, A. 2003. Functional expression cloning of Nanog, a pluripotency sustaining factor in embryonic stem cells. Cell 113: 643-655.

Ernst, P., Mabon, M., Davidson, A.J., Zon, L.I., and Korsmeyer, S.J. 2004. An Mll-dependent Hox program drives hematopoietic progenitor expansion. Curr. Biol. 14: 2063-2069.

Glaser, S., Schaft, J., Lubitz, S., Vintersten, K., van der Hoeven, F., Tufteland, K.R., Aasland, R., Anastassiadis, K., Ang, S.L., and Stewart, A.F. 2006. Multiple epigenetic maintenance factors implicated by the loss of Mll2 in mouse development. Development 133: 1423-1432.

Houlard, M., Berlivet, S., Probst, A.V., Quivy, J.P., Hery, P., Almouzni, G., and Gerard, M. 2006. CAF-1 is essential for heterochromatin organization in pluripotent embryonic cells. PLoS Genet. 2: e181. doi: 10.1371/journal.pgen.0020181.

Issaeva, I., Zonis, Y., Rozovskaia, T., Orlovsky, K., Croce, C.M., Nakamura, T., Mazo, A., Eisenbach, L., and Canaani, E. 2007. Knockdown of ALR (MLL2) reveals ALR target genes and leads to alterations in cell adhesion and growth. Mol. Cell. Biol. 27: 1889-1893.

Ivanova, N., Dobrin, R., Lu, R., Kotenko, I., Levorse, J., DeCoste, C., Schafer, X., Lun, Y., and Lemischka, I.R. 2006. Dissecting self-renewal in stem cells with RNA interference. Nature 442: 533-538.

Kaji, K., Nichols, J., and Hendrich, B. 2007. Mbd3, a component of the NuRD co-repressor complex, is required for development of pluripotent cells. Development 134: 1123-1132.

Klose, R.J. and Zhang, Y. 2007. Regulation of histone methylation by demethylimination and demethylation. Nat. Rev. Mol. Cell Biol. 8: 307-318.

Klose, R.J., Yamane, K., Bae, Y., Zhang, D., Erdjument-Bromage, H., Tempst, P., Wong, J., and Zhang, Y. 2006. The transcriptional repressor JHDM3A demethylates trimethyl histone H3 lysine 9 and lysine 36. Nature 442: 312-316.

Knoepfler, P.S., Zhang, X.Y., Cheng, P.F., Gafken, P.R., McMahon, S.B., and Eisenman, R.N. 2006. Myc influences global chromatin structure. EMBO J. 25: 2723-2734.

Lan, F., Bayliss, P.E., Rinn, J.L., Whetstine, J.R., Wang, J.K., Chen, S., Iwase, S., Alpatov, R., Issaeva, I., Canaani, E., et al. 2007. A histone H3 lysine 27 demethylase regulates animal posterior development. Nature doi: 10.1038/nature06192.

Lee, T.I., Jenner, R.G., Boyer, L.A., Guenther, M.G., Levine, S.S., Kumar, R.M., Chevalier, B., Johnstone, S.E., Cole, M.F., Isono, K., et al. 2006. Control of developmental regulators by Polycomb in human embryonic stem cells. Cell 125: 301313.

Lin, W., Srajer, G., Evrard, Y.A., Phan, H.M., Furuta, Y., and Dent, S.Y. 2007. Developmental potential of Gen5 $5^{-/-}$embryonic stem cells in vivo and in vitro. Dev. Dyn. 236: 15471557. 
Loh, Y.H., Wu, Q., Chew, J.L., Vega, V.B., Zhang, W., Chen, X., Bourque, G., George, J., Leong, B., Liu, J., et al. 2006. The Oct4 and Nanog transcription network regulates pluripotency in mouse embryonic stem cells. Nat. Genet. 38: 431440.

Loh, Y.-H., Zhang, W., Chen, X., Grorge, J., and Ng, H.-H. 2007. Jmjd1a and Jmjd2c histone $\mathrm{H} 3$ Lys 9 demethylases regulate self-renewal in embryonic stem cells. Genes \& Dev. 21: 2545-2557.

Maherali, N., Sridharan, R., Xie, W., Utikal, J., Eminli, S., Arnold, K., Stadtfeld, M., Yachechko, R., Tchieu, J., Jaenisch, R., et al. 2007. Directly reprogrammed fibroblasts show grobal epigenetic remodeling and widespread tissue contribution. Cell Stem Cell 1: 55-70.

Masui, S., Nakatake, Y., Toyooka, Y., Shimosato, D., Yagi, R., Takahashi, K., Okochi, H., Okuda, A., Matoba, R., Sharov, A.A., et al. 2007. Pluripotency governed by Sox 2 via regulation of Oct $3 / 4$ expression in mouse embryonic stem cells. Nat. Cell Biol. 9: 625-635.

Matoba, R., Niwa, H., Masui, S., Ohtsuka, S., Carter, M.G. Sharov, A.A., and Ko, M.S. 2006. Dissecting Oct3/4regulated gene networks in embryonic stem cells by expression profiling. PLOS ONE 1: e26. doi: 10.1371/journal.pone. 0000026.

Meshorer, E., Yellajoshula, D., George, E., Scambler, P.J., Brown, D.T., and Misteli, T. 2006. Hyperdynamic plasticity of chromatin proteins in pluripotent embryonic stem cells. Dev. Cell 10: 105-116.

Mitsui, K., Tokuzawa, Y., Itoh, H., Segawa, K., Murakami, M., Takahashi, K., Maruyama, M., Maeda, M., and Yamanaka, S. 2003. The homeoprotein Nanog is required for maintenance of pluripotency in mouse epiblast and ES cells. Cell 113: 631-642.

Montgomery, N.D., Yee, D., Chen, A., Kalantry, S., Chamberlain, S.J., Otte, A.P., and Magnuson, T. 2005. The murine polycomb group protein Eed is required for global histone $\mathrm{H} 3$ lysine-27 methylation. Curr. Biol. 15: 942-947.

Nakatake, Y., Fukui, N., Iwamatsu, Y., Masui, S., Takahashi, K., Yagi, R., Yagi, K., Miyazaki, J.I., Matoba, R., Ko, M.S., et al. 2006. Klf4 cooperates with Oct3/4 and Sox 2 to activate the Lefty1 core promoter in embryonic stem cells. Mol. Cell. Biol. 26: 7772-7782.

Narducci, M.G., Fiorenza, M.T., Kang, S.M., Bevilacqua, A., Di Giacomo, M., Remotti, D., Picchio, M.C., Fidanza, V., Cooper, M.D., Croce, C.M., et al. 2002. TCL1 participates in early embryonic development and is overexpressed in human seminomas. Proc. Natl. Acad. Sci. 99: 11712-11717.

Niwa, H. 2007. How is pluripotency determined and maintained? Development 134: 635-646.

Niwa, H., Toyooka, Y., Shimosato, D., Strumpf, D., Takahashi, K., Yagi, R., and Rossant, J. 2005. Interaction between Oct3/4 and Cdx2 determines trophectoderm differentiation. Cell 123: 917-929.

Ooi, S.K., Qiu, C., Bernstein, E., Li, K., Jia, D., Yang, Z., Erdjument-Bromage, H., Tempst, P., Lin, S.P., Allis, C.D., et al. 2007. DNMT3L connects unmethylated lysine 4 of histone H3 to de novo methylation of DNA. Nature 448: 714717.

Pasini, D., Bracken, A.P., Hansen, J.B., Capillo, M., and Helin, K. 2007. The polycomb group protein Suz12 is required for embryonic stem cell differentiation. Mol. Cell. Biol. 27: 37693779.

Sumi-Ichinose, C., Ichinose, H., Metzger, D., and Chambon, P. 1997. SNF2 $\beta$-BRG1 is essential for the viability of F9 murine embryonal carcinoma cells. Mol. Cell. Biol. 17: 5976-5986.

Szutorisz, H., Georgiou, A., Tora, L., and Dillon, N. 2006. The proteasome restricts permissive transcription at tissue-specific gene loci in embryonic stem cells. Cell 127: 1375-1388.

Tada, M., Takahama, Y., Abe, K., Nakatsuji, N., and Tada, T. 2001. Nuclear reprogramming of somatic cells by in vitro hybridization with ES cells. Curr. Biol. 11: 1553-1558.

Takahashi, K. and Yamanaka, S. 2006. Induction of pluripotent stem cells from mouse embryonic and adult fibroblast cultures by defined factors. Cell 126: 663-676.

Takeuchi, T., Yamazaki, Y., Katoh-Fukui, Y., Tsuchiya, R., Kondo, S., Motoyama, J., and Higashinakagawa, T. 1995 Gene trap capture of a novel mouse gene, jumonji, required for neural tube formation. Genes \& Dev. 9: 1211-1222.

Teitell, M.A. 2005. The TCL1 family of oncoproteins: Co-activators of transformation. Nat. Rev. Cancer 5: 640-648.

Tsukada, Y., Fang, J., Erdjument-Bromage, H., Warren, M.E., Borchers, C.H., Tempst, P., and Zhang, Y. 2006. Histone demethylation by a family of JmjC domain-containing proteins. Nature 439: 811-816.

Yamanaka, S. 2007. Strategies and new developments in the generation of patient-specific pluripotent stem cells. Cell Stem Cell 1: 39-50.

Yamane, K., Toumazou, C., Tsukada, Y., Erdjument-Bromage, H., Tempst, P., Wong, J., and Zhang, Y. 2006. JHDM2A, a JmjC-containing H3K9 demethylase, facilitates transcription activation by androgen receptor. Cell 125: 483-495.

Yuan, H., Corbi, N., Basilico, C., and Dailey, L. 1995. Developmental-specific activity of the FGF-4 enhancer requires the synergistic action of Sox 2 and Oct-3. Genes \& Dev. 9: 26352645. 


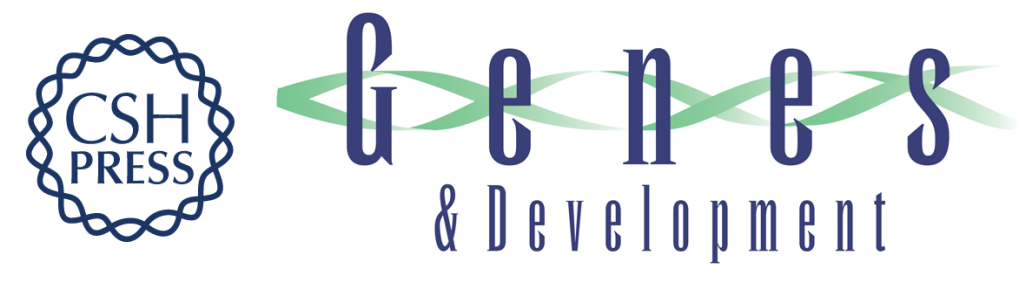

\section{Open conformation chromatin and pluripotency}

Hitoshi Niwa

Genes Dev. 2007, 21:

Access the most recent version at doi:10.1101/gad.1615707

\section{Related Content Jmjd1a and Jmjd2c histone H3 Lys 9 demethylases regulate self-renewal in embryonic stem cells \\ Yuin-Han Loh, Weiwei Zhang, Xi Chen, et al. \\ Genes Dev. October , 2007 21:2545-2557 \\ References This article cites 39 articles, 12 of which can be accessed free at: \\ http://genesdev.cshlp.org/content/21/21/2671.full.html\#ref-list-1 \\ Articles cited in: \\ http://genesdev.cshlp.org/content/21/21/2671.full.html\#related-urls \\ License \\ Email Alerting \\ Receive free email alerts when new articles cite this article - sign up in the box at the top \\ Service right corner of the article or click here.}

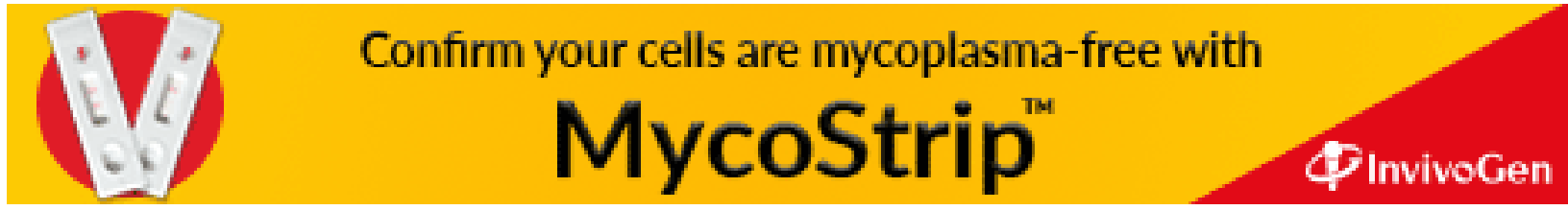

